\title{
Total artificial heart: patient selection and risk factors
}

\author{
Thomas Senage $^{1,2}$, Charles-Henri David ${ }^{2}$, Prakash Nanjaiah ${ }^{1}$, Jean-Christian Roussel $^{2}$ \\ ${ }^{1}$ Royal Papworth Hospital NHS Foundation Trust, Cambridge, UK; ${ }^{2}$ Department of Thoracic and CardioVascular Surgery, Thorax Institut, \\ University of Nantes, Nantes, France \\ Correspondence to: Jean-Christian Roussel. Chirurgie Thoracique et Cardiovasculaire, Institut du Thorax, Boulevard Jacques Monod, 44093 Nantes \\ Cedex 1, France. Email: jeanchristian.roussel@chu-nantes.fr.
}

Submitted Dec 10, 2019. Accepted for publication Feb 04, 2020.

doi: $10.21037 /$ acs.2020.02.10

View this article at: http://dx.doi.org/10.21037/acs.2020.02.10

The patient selection for long-term mechanical circulatory assistance is based on the objective to be achieved, inclusive of bridge to transplantation, destination therapy, or bridge to decision. In case of biventricular failure, the total artificial heart offers a reliable solution in bridge to transplantation (1), with excellent long-term results. However, appropriate patient selection is still essential (2). This editorial focuses on the relevant factors to be considered for selecting suitable patients.

\section{How to confirm biventricular failure?}

Apart from the context of malignant arrhythmias or intraventricular thrombi, one of the first difficulties concerns the confirmation of the diagnosis of irreversible failure of both ventricles, particularly that of the right ventricle. Indirect criteria, such as imaging, biochemical parameters and patient hemodynamic status, can sometimes yield misleading diagnoses and a right ventricle that seems "sufficiently functional" in presence of concomitant left ventricular failure may reveal its dysfunction after restoration of left ventricular function. There are many scores and algorithms in the literature that predict this right ventricular failure with varying accuracy (3), which ultimately represents our lack of knowledge about the right ventricle. A significant number of LVAD patients will end up with right ventricular failure, unfortunately some of them permanent. In case of such borderline unpredictable situations, some teams now propose to implement a transient left ventricular assistance prior to implanting the TAH, in order to unmask this right ventricular failure (4).
Once the double ventricular failure is confirmed, which patient will benefit from implanting a total artificial heart (TAH)?

After comprehensive analysis of many patient risk factors for complications, several teams have surprisingly revealed that smoking history was the strongest independent risk factor after post-TAH transplantation $(2,5)$. Age factor also needs consideration, since the vast majority of patients implanted will be on the bridge to transplantation. Age greater than 55 in itself is not prohibitive but we must strongly debate the merits of implanting a TAH in such a cohort of patients $(1,3)$. However, assessing the physiological age of the patient would be more valuable rather than the actual age number. A higher fragility index and/or severe malnutrition guarantees the occurrence of post-implantation complications (i.e., infection, bleeding, diffuse cavitary effusions, prolonged ventilatory support, tracheostomy, prolonged hemofiltration, stroke, etc.) and essentially leads to a long and difficult rehabilitation.

There are fewer absolute contraindications to TAH implant (life expectancy of less than 2 years or malignancy), as compared to those for routine heart transplantation, due to the ability of the TAH to reverse the multiorgan dysfunction in these patients. However, there are a few relative contraindications which can lead to a greater incidence of complications. A history of prior cardiac surgery has been considered as an independent mortality risk factor in many studies $(1,3)$. In such patients, we would logically proceed to heart transplantation without going through the total artificial heart stage. There is a significant risk of major bleeding in this patient cohort because of the need for higher effective anticoagulation for its four mechanical 
valves. There is also an increased risk of infections as well as higher pericardial inflammation and its sequelae.

In cases of pre-existing right ventricular dysfunction, liver and kidney impairment have been extensively analyzed in context of the TAH series, without any improvement in the ability to predict their evolution under assistance (6-8). In addition, TAH has shown us its ability to re-establish both a satisfactory organ perfusion and adequate venous return that can reverse liver and/or kidney failure. Neither hepatic parenchymal damage as evidenced by abnormal liver function tests nor renal failure was found to be a risk factor for death (1,3-5). Nevertheless, we arbitrarily set ourselves a maximum threshold for bilirubinemia which, combined with an upward trend, led us to believe that the necrosis of the bile ducts would then be probably irreversible.

The presence of thrombophilia is an absolute contraindication for TAH with mechanical valves. However, the implantation of a "biocompatible" artificial heart such as the Carmat ${ }^{\circledR}$ would remove this contraindication (9).

Finally, severe psychosocial limitations and medical non-adherence should remain absolute contraindications, as when implanting a mono-ventricular device (10). For example, psychotic patients with body dysmorphic disorders are unable to accept the pump implantation resulting in non-compliance with treatment and therefore ultimately leading to failure with therapy.

With regards to device-related contraindications, it is important to consider the size and body habitus of the patient. The typical patient for TAH is a man with a body surface area greater than $1.8 \mathrm{~m}^{2}$. Although implantation in women or in patients with a smaller body surface area is often possible, it is more complicated due to the large size of the TAH. The recent introduction of smaller artificial ventricles to the market has helped to alleviate some of this size incompatibility but it is associated with a reduction in the overall cardiac output of the device, which becomes inadequate in cases of obese patients with reduced thoracic cavity volume and specific clinical situation requiring high cardiac output states like sepsis. In borderline cases, the size of the artificial heart to be implanted is most often planned by the CT-scan guided measurement of the thoracic anterior-posterior distance, or more recently using $3 \mathrm{D}$ reconstruction of the patient's thorax with virtual fitting of the implanted device (11).

\section{Conclusions}

The total artificial heart has now largely proven its effectiveness in managing complicated patients with double ventricular failure. However, knowledge of above mentioned contraindications and the impact of highrisk situations is essential for a good selection of potential patients.

\section{Acknowledgments}

None.

\section{Footnote}

Conflicts of Interest: The authors have no conflicts of interest to declare.

\section{References}

1. Arabía FA, Cantor RS, Koehl DA, et al. Interagency registry for mechanically assisted circulatory support report on the total artificial heart. J Heart Lung Transplant 2018;37:1304-12.

2. Roussel JC, Baron O, Périgaud C, et al. Outcome of heart transplants 15 to 20 years ago: graft survival, posttransplant morbidity, and risk factors for mortality. J Heart Lung Transplant 2008;27:486-93.

3. Fida N, Loebe M, Estep JD, et al. Predictors and management of right heart failure after left ventricular assist device implantation. Methodist Debakey Cardiovasc J 2015;11:18-23.

4. Lima B, Kale P, Gonzalez-Stawinski GV, et al. Effectiveness and Safety of the Impella 5.0 as a Bridge to Cardiac Transplantation or Durable Left Ventricular Assist Device. Am J Cardiol 2016;117:1622-8.

5. Remadi JP, Baron O, Roussel JC, et al. Myocardial preservation using celsior solution in cardiac transplantation: early results and 5-year followup of a multicenter prospective study of 70 cardiac transplantations. Ann Thorac Surg 2002;73:1495-9.

6. Copeland JG, Smith RG, Bose RK, et al. Risk factor analysis for bridge to transplantation with the CardioWest total artificial heart. Ann Thorac Surg 2008;85:1639-44.

7. Roussel JC, Sénage T, Baron O, et al. CardioWest (Jarvik) total artificial heart: a single-center experience with 42 patients. Ann Thorac Surg 2009;87:124-9; discussion 130.

8. Kirsch ME, Nguyen A, Mastroianni C, et al. SynCardia Temporary Total Artificial Heart as Bridge to Transplantation: Current Results at La Pitié Hospital. Ann Thorac Surg 2013;95:1640-6. 
9. Latrémouille C, Carpentier A, Leprince P, et al. A bioprosthetic Total Artificial Heart for end-stage heart failure: results from the pilot study. J Heart Lung Transplant 2018;37:33-7.

10. Lund LH, Matthews J, Aaronson K. Patient selection for left ventricular assist devices. Eur J Heart Fail

Cite this article as: Senage T, David CH, Nanjaiah P, Roussel JC. Total artificial heart: patient selection and risk factors. Ann Cardiothorac Surg 2020;9(2):118-120. doi: 10.21037/ acs.2020.02.10
2010;12:434-43.

11. Fritschi AJ, Laumen M, Spiliopoulos S, et al. Image based evaluation of mediastinal constraints for the development of a pulsatile total artificial heart. Biomed Eng Online $2013 ; 12: 81$. 